\title{
Modelo matemático entre el puntaje de examen de ingreso y el rendimiento académico de los estudiantes ingresantes a la Universidad Nacional Jorge Basadre Grohmann, año académico 2018
}

\author{
Mathematical model between the examination exam score and academic \\ performance of students entering the National University Jorge Basadre Grohmann, \\ academic year 2018
}

${ }^{11}$ Humberto Vargas Pichon; ${ }^{2}$ Luis Solorzano Espinola; ${ }^{3}$ Wilson Chanini Choquecota.

\section{RESUMEN}

Tipo de estudio: No-experimental. Objetivo general: Determinar en qué medida un modelo matemático explica la relación entre el puntaje de examen de ingreso y el rendimiento académico de los estudiantes ingresantes a la Universidad Nacional Jorge Basadre Grohmann, año académico 2018. Población y muestra: La población comprendió 1664 estudiantes y la muestra conformada por 1551 estudiantes cuya selección fue por conveniencia (no se consideró a los estudiantes que ingresaron, pero no se matricularon y los que habiéndose matriculado se retiraron). Resultado: Existe una relación directa y significativa entre el puntaje examen de ingreso y el rendimiento académico de los estudiantes ingresantes de la Universidad Nacional Jorge Basadre Grohmann, año académico 2018. Dando el siguiente modelo matemático $\mathrm{y}=6.289+0.015 \mathrm{x}$, con $\mathrm{R}$ cuadrado $=0.145$.

Palabras Claves: Puntaje de examen de ingreso, rendimiento académico

\begin{abstract}
Type of study: not experimental. Course objective: To determine to what extent a mathematical model explains the relationship between the entrance exam score and the academic performance of the students admitted to the Jorge Basadre Grohmann National University, academic year 2018. Population and sample: The population comprised 1664 students and the sample made up of 1551 students whose selection was for convenience (Students who entered, but were not enrolled, were not considered those who had enrolled withdrew). Result: There is a direct and significant relationship between the entrance exam score and the academic performance of the incoming students of the Jorge Basadre Grohmann National University, academic year 2018. Giving the following mathematical model $\mathrm{y}=6,289+0,015 \mathrm{x}$, with $\mathrm{R}$ square $=0,145$.
\end{abstract}

Keywords: Entrance exam score, academic performance.

\section{INTRODUCCIÓN}

Fortalecer el capital humano en el Perú, mediante la educación superior, resulta prioritario para el desarrollo en un entorno de competitividad. Por tal motivo, se debe lograr que el mayor número de jóvenes egresados de la educación básica regular acceda y culmine satisfactoriamente la educación superior, logrando una masa crítica de profesionales que se inserten adecuadamente en un mercado laboral cada vez más competitivo. En ese sentido, una educación más eficiente y de mejor calidad que promueva la equidad de oportunidades y fomente un alto rendimiento académico, será cada vez más necesaria para formar profesionales competitivos que el país necesita para impulsar su desarrollo.
Para lograr una permanencia efectiva de los estudiantes en las universidades, es necesario analizar las causas del rendimiento académico de los estudiantes universitarios en los primeros años de estudio, en particular en las asignaturas críticas, donde existe un alto número de desaprobación.

La literatura especializada coincide en que son muchos los factores que influyen en el rendimiento académico: factores de identificación, factores psicológicos, factores socio-familiares, factores psicológicos y factores socioeconómicos, etc., que pueden impactar fuertemente en |59los logros del aprendizaje. Sin embargo, existe un consenso general en que el rendimiento académico previo (diferentes formas de examen de ingreso en la universidad: examen de ingreso ordinario, examen de ingreso extraordinario y

\footnotetext{
'Universidad Nacional Jorge Basadre Grohmann. Tacna - Perú E-mail: humberto.vargas@unjbg.edu.pe

${ }^{2}$ Universidad Nacional Jorge Basadre Grohmann. Tacna - Perú E-mail: 1solorzanoe@hotmail.com

${ }^{3}$ Universidad Nacional Jorge Basadre Grohmann. Tacna - Perú E-mail: Wilson_chanini@hotmail.com
} 
Centro Pre Universitario (CEPU), es la mejor variable para predecir el rendimiento académico posterior.

\section{Contexto mundial}

El acceso a la educación universitaria selectiva en Chile ordena a los postulantes de acuerdo a un puntaje que pondera los factores de Trayectoria Escolar y Prueba de Selección Universitaria (PSU). En 2013, la inclusión del Ranking de Notas ocasionó una reestructuración en la ponderación de los factores de selección, redefiniendo las posiciones institucionales al respecto. Con bases de datos oficiales y procedimientos estadísticos ad hoc, se describe el comportamiento de los 1423 programas de pregrado ofertados por las 33 universidades integrantes del sistema único de admisión en 2014. Se observa que el componente PSU pierde hegemonía comparado con la trayectoria escolar como factor de ponderación, resignificándose especialmente el ranking. Esta es una tendencia generalizada, pero de comportamiento no homogéneo. Posiciones institucionales escindidas en la valoración del desempeño escolar, a partir del nivel de selectividad tradicional del programa, evidencian estrategias diferenciadas de reclutamiento en una gradiente de adhesión a políticas pro inclusión y defensa de intereses institucionales (Rodríguez \& Padilla, 2016, p. 313).

Si bien el rendimiento académico del estudiantado que ingresa a las universidades chilenas ha sido objeto de múltiples estudios, pocas investigaciones han analizado la relación que tienen los instrumentos de selección de ingreso y el rendimiento académico posterior. En una muestra de 440 estudiantes de Ingeniería Comercial de la Universidad Austral de Chile, pertenecientes a cinco promociones de primer año, se estudiaron los instrumentos de admisión a la carrera, y se determinó cuáles explican mejor el rendimiento académico. Para ello se utilizaron los resultados de las PSU y las Notas de Enseñanza Media (NEM) obtenidos por el estudiantado, en relación con el Promedio Semestral Ponderado (PSP) del primer año universitario. Se determinó que el promedio de NEM explica mejor el rendimiento académico estudiantil, al presentar la mejor correlación con el rendimiento académico del primer semestre y de manera más fuerte en el segundo. (Vergara \& Peredo, 2017, p. 95)

\section{Contexto nacional}

Tenemos la investigación, en Lima cuyo título es: "Relación entre las modalidades de ingreso y el rendimiento académico de los estudiantes de estomatología de las cohortes 2008-2009 en una Universidad Privada".

Objetivos: Determinar la relación entre las modalidades de ingreso y el rendimiento académico universitario de los estudiantes de estomatología de las cohortes 2008-2009 de la Universidad Peruana Cayetano Heredia (UPCH). Material y métodos: Diseño de corte descriptivo, observacional, longitudinal y retrospectivo, se realizó en la población total de estudiantes de la Facultad de Estomatología Roberto Beltrán de la UPCH que ingresaron en los años
2008 y 2009 que cursaron el primer y segundo año académico. En el 2008 ingresaron 118 y en el 2009 fueron 110. Resultados: Respecto a las modalidades de ingreso se observa que el mayor número de estudiantes ingresaron por Factor Excelencia $(\mathrm{N}=135$, $59 \%)$, seguido de Examen de admisión ( $\mathrm{N}=52,22 \%)$ y Centro Preuniversitario-CEPREU ( $\mathrm{N}=38,16 \%)$, respecto al rendimiento académico universitario según género se encontró que el mayor número de estudiantes fueron de sexo femenino $(\mathrm{N}=159,70 \%)$ seguido del sexo masculino $(\mathrm{N}=69,30 \%)$, no se encontró diferencias estadísticamente significativas respecto al rendimiento académico universitario según sexo. Conclusiones: Respecto al estudio de la relación del rendimiento académico universitario con las modalidades de ingreso se encontró que los estudiantes obtuvieron promedios similares en el rendimiento académico universitario al margen de la modalidad de ingreso, en términos de promedio ponderado anual, la relación no fue estadísticamente significativa $(\mathrm{P}>0,05)$. Las modalidades de admisión parecen no ser un buen predictor del rendimiento académico; salvo el rendimiento escolar previo, una vez que el estudiante ingresa a la universidad se disipan algunas variables que los caracterizan durante la etapa escolar. (Flores, 2012, p. 210).

Asimismo, se tiene la investigación cuyo objetivo principal fue: establecer la relación entre las habilidades sociales y el clima social familiar con el rendimiento académico en un grupo de estudiantes universitarios de Lima Metropolitana. Para su realización se evaluó a 205 estudiantes, de ambos sexos, mayores de 16 años, que cursaban el I ciclo de estudios en Psicología, procedentes de Universidad Particular de San Martín de Porres y de Universidad Nacional Mayor de San Marcos, a quienes se aplicó la Lista de Chequeo de Habilidades Sociales de Goldstein, traducida y adaptada a nuestro país por Ambrosio Tomás entre 1994 - 95 y la escala de clima social en la familia de Moos y Trickett estandarizada en el Perú por Ruiz y Guerra en 1993. Los resultados de la investigación fueron procesados por el coeficiente de correlación de pearson, prueba chi cuadrado, media y desviación estándar, el coeficiente alfa de cronbach. Los resultados arrojaron una correlación positiva y significativa entre habilidades sociales y el clima social en la familia, encontrándose también que no existe estadísticamente una correlación significativa entre habilidades sociales y clima social en la familia con el rendimiento académico; se encontró además que en relación con las variables habilidades sociales y clima social en la familia más del $50 \%$ de los alumnos de la muestra total se ubicaron en el nivel promedio $(65.9 \%$ y $62.7 \%$ respectivamente) y en rendimiento académico solo un porcentaje bajo de alumnos (9.8\%) obtuvo notas desaprobatorias (García, 2005, p. 63).

\section{Contexto local}

Tenemos la Investigación: Rendimiento académico de ingreso y su relación con el rendimiento académico en el primer año de educación universitaria de la UNJBG en año 2006. El objetivo de la presente investigación es determinar el grado de relación que existe entre el rendimiento académico de secundaria con el rendimiento académico alcanzado por el estudiante en el primer año de educación universitaria en la 
Universidad Nacional Jorge Basadre Grohmann de Tacna, en el año 2006. El método es empírico, basado en análisis documental de rendimiento de ingreso y rendimiento alcanzado por los estudiantes según actas finales de evaluación. Las conclusiones a las que se arribó al finalizar esta investigación son: 1) El rendimiento académico de los estudiantes de secundaria al ingresar a la universidad se concentra en la categoría de regular a bueno, pues no hay desaprobados, con un $56.3 \%$ (regular) y un $43.1 \%$ (bueno). 2) El rendimiento académico de los estudiantes en el primer año de estudios universitarios se concentra en la categoría de regular con un $49.7 \%$ (regular) y un $17.9 \%$ (bueno). 3) El 32,4\% de estudiantes disminuyen su rendimiento académico en el primer año de universidad y pasan de la condición de aprobados al ingresar a la universidad a la condición de desaprobados (Chávez \& Mendoza, 2006, p. 47).

También, se tiene la Investigación Relación entre el examen de admisión y el académico, 2001-2005. El abandono y la prolongación de los estudios constituyen en la actualidad un importante problema en la Universidad. En el presente informe se presentan los resultados de un estudio transversal retrospectivo que tenía por objetivo establecer la relación entre el puntaje del examen de ingreso y el rendimiento académico de los ingresantes del 2001 a la Facultad de Ciencias de la Educación de la UNJBG. Se determinó que existe relación directa $(\mathrm{p}<0,05)$ con una baja correlación $(\mathrm{r}=0,34)$, esto, con escaso significado estadístico (Benites \& Ticona, 2006, p. 15).

\section{Sobre exámenes de admisión en las universidades}

La educación superior se ha visto enfrentada a retos sin precedente desde inicios del siglo XXI, bajo el impacto de la globalización y el crecimiento económico, además de la revolución de la información y la comunicación. Estos cambios trascendentales han obligado a las instituciones a mejorar la calidad de la educación que ofrecen, por lo que se han replanteado los mecanismos de selección de los aspirantes, para que, a través de esta selección, se garantice la formación de egresados de la más alta calidad académica que sean competitivos para ocupar los reducidos espacios que ofrece el mercado laboral (Cortés \& Palomar 2008, p. 200). Para asegurar la calidad de la educación superior, se debe partir de la selección de los estudiantes considerando los factores que influyen sobre el rendimiento académico y que repercuten en la deserción estudiantil sobre todo durante la transición del colegio a la universidad. La universidad sólo debe admitir como estudiantes a aquellas personas que tengan un legítimo y autentico interés por el conocimiento como factor necesario para el avance social (Flores, 2012, p. 211).

El objetivo de los procesos de selección es obtener información de un conjunto de examinados sobre algún dominio específico, como habilidades o conocimientos, mediante la asignación de puntajes. Un ejemplo de este tipo de procesos es el ingreso a la educación superior, en el que se utiliza una serie de instrumentos que permiten tomar decisiones sobre los participantes y las carreras o universidades a las que postulan. Así, el diseño apropiado de los mecanismos de selección es clave, ya que si estos no son adecuados pueden generar un significativo perjuicio para los postulantes, ya que tarde o temprano pueden fracasar en sus estudios (Larroucau et al, 2015, p. 96).

En primer lugar, basándose en las tradicionales pruebas de ingreso, todos los puntajes de los solicitantes están siendo comparados con el mismo criterio, sin embargo, el contenido de los exámenes puede estar sesgado en contra de ciertos grupos, por ejemplo, si se hace uso de conocimientos o experiencias a los que una cultura o grupos específicos de ella no han tenido acceso, o con criterios subjetivos que no se comparten en la intersubjetividad de examinadores y grupos de examinados. De esta forma, realizar evaluaciones basadas solamente en las calificaciones de los exámenes no proporciona una comparación equitativa de los méritos reales de los solicitantes. Por lo tanto, puede argumentarse que los procesos de admisión que tengan en cuenta solo los resultados de los exámenes, aunque tal vez sean relativamente objetivos, no son en realidad equitativos.

Claramente, las instituciones de educación superior, en su afán de mejorar su calidad, se ven obligadas a definir adecuados instrumentos en su proceso de selección de estudiantes, como se cita anteriormente, considerando diversos criterios: académicos, políticos, sociales, económicas, culturales, entre otros.

\section{Sobre rendimiento académico en las universidades}

Para el desarrollo de un país es de gran importancia la formación y calificación de su capital humano. En ese sentido las universidades juegan un rol muy importante ya que son las responsables de la formación de profesionales, junto a las instituciones de educación superior técnico-profesional e institutos pedagógicos. Cada vez más se incrementa el interés de las universidades y de los gobiernos por el rendimiento académico y, especialmente, por el abandono de los estudios, como se evidencia en las políticas de estado en relación a evaluar la calidad de las universidades. Tal es el caso del Perú, que en 2014 promulgó una nueva Ley Universitaria, crea la Superintendencia Nacional de Educación Superior Universitaria (SUNEDU) y se establece el licenciamiento obligatorio y renovable de las universidades, en lugar de la autorización de funcionamiento del anterior marco legal. Posteriormente, se aprobó la Política de Aseguramiento de la Calidad de la Educación Superior Universitaria la misma que establece el proceso de licenciamiento obligatorio como uno de los cuatro pilares del Sistema de Aseguramiento de la Calidad (https://www.sunedu.gob.pe).

Las tendencias de la educación superior, han obligado a estas a diseñar estrategias, que permitan incrementar la calidad de la formación y a evaluar constantemente su quehacer, con la intención de lograr un mejor rendimiento académico en los estudiantes y su permanencia hasta lograr terminar sus estudios profesionales en un periodo adecuado.

El rendimiento académico alude al promedio de calificaciones obtenido por el alumno, en las asignaturas, en las cuales, ha presentado examen, independientemente del tipo de examen. Su indicador es la suma total de calificaciones obtenidas, divididas 
entre el número de calificaciones. La autora continúa afirmando que el rendimiento escolar o académico es el nivel de conocimientos demostrado en un área o materia, comparado con la norma (edad, nivel académico) y las calificaciones suelen reflejar las preferencias, la dedicación y la capacidad mayor o menor de los alumnos en distintas materias (TreviñoLozano, 2003, pp. 29-30).

\section{MATERIALES Y MÉTODOS}

Enfoque metodológico: La investigación centró el desarrollo de su metodología dentro del paradigma positivista, bajo un enfoque cuantitativo, de tipo aplicada, correlacional pues demuestra la relación que existe entre ambas variables de estudio, y de diseño no experimental, descriptivo, correlacional, predictivo y retrospectivo.

Variables de estudio: Puntaje de Examen de Ingreso y Rendimiento académico.

La población comprendió 1664 estudiantes ingresantes y matriculados en la Universidad Nacional Jorge Basadre Grohmann (UNJBG) correspondiente al año académico 2018. La muestra estuvo conformada por 1551 estudiantes, miembros de la población antes mencionada cuya selección fue dada por conveniencia (no se consideró a los estudiantes que ingresaron, pero no se matricularon y los que habiéndose matriculado se retiraron).
Instrumentos de recolección de datos: Para la variable Puntaje de examen de ingreso se usó la técnica del análisis documental y como instrumento los registros de notas de la Oficina de admisión y/o Centro Pre Universitario (CEPU). Para la variable Rendimiento Académico se utilizó la Técnica del Análisis documental y como instrumento el ranking académico de los estudiantes ingresantes a la Universidad Nacional Jorge Basadre Grohmann, año académico 2018 (DASA-UNJBG).

Validación de instrumentos: Según la información recabada de la Dirección Académica de actividades y Servicios Académicos (DASA) y el Ranking Académico UNJBG 2018.

Validación de las escalas de valores utilizadas: Según criterio de los investigadores.

Análisis estadístico y presentación de los datos: Estadística descriptiva: Mínimo, máximo, media y desviación estándar.

Estadística inferencial: Análisis inferencial cuantitativo

Se hizo usando la correlación de Pearson y regresión lineal, con un nivel de significancia de $=0,05$ y con una confiabilidad del $95 \%$.

\section{RESULTADOS}

Usando el software estadístico SPSS versión 20 se obtuvo los siguientes resultados:

Tabla 01: Estadísticos descriptivos de las variables Puntaje de Examen de Ingreso y Rendimiento académico de los estudiantes ingresantes a la Universidad Nacional Jorge Basadre Grohmann, año académico 2018

$\begin{array}{lcrrrr} & \text { N } & \text { Mínimo } & \text { Máximo } & \text { Media } & \text { Desv. típ. } \\ \text { Puntaje de } & 1551 & 80,0 & 580,5 & 294,859 & 94,2637 \\ \text { Examen } & & & & & \\ \begin{array}{l}\text { Rendimiento académico } \\ 2018\end{array} & 1551 & 0,091 & 17,591 & 10,67460 & 3,680532 \\ \text { N válido (según lista) } & 1551 & & & & \end{array}$

Fuente: matriz de sistematización de datos con SPSS, setiembre 2019

El promedio del puntaje de examen de ingreso de los estudiantes ingresantes a la Universidad Nacional Jorge Basadre Grohmann, año académico 2018, fue de 294,859. Teniendo en cuenta como puntaje mínimo de ingreso de 80,0; y el máximo de 580,5. y teóricamente el promedio de los alejamientos de los datos respecto a la media (desviación estándar) fue de 94.2637. Por otro lado, el promedio del Rendimiento Académico de los estudiantes ingresantes a la Universidad Nacional Jorge Basadre Grohmann, año académico 2018, fue de 10.674 60. Teniendo en cuenta como el mínimo Rendimiento académico de 0.091 y el máximo de 17.591 y teóricamente el promedio de los alejamientos de los datos respecto a la media (desviación estándar) fue de 3.68 . 
Modelo matemático entre el puntaje de examen de ingreso y el rendimiento académico de los estudiantes ingresantes a la Universidad Nacional Jorge Basadre Grohmann, año académico 2018

Tabla 02. Estadísticos Inferencial (correlación Pearson) de las variables de Puntaje de Examen de Ingreso y el Rendimiento Académico de los estudiantes ingresantes a la Universidad Nacional Jorge Basadre Grohmann, año académico 2018 .

$\begin{array}{llrr} & & \text { Puntaje de } & \text { Rendimiento académico } \\ \text { Euntaje de } & \text { Correlación de Pearson } & 1 & 2018 \\ \text { Examen } & \text { Sig. (bilateral) } & & 0,381^{* *} \\ & \mathrm{~N} & 1551 & 0,000 \\ \text { Rendimiento académico } 2018 & \text { Correlación de Pearson } & 0,381^{* *} & 1551 \\ & \text { Sig. (bilateral) } & 0,000 & 1 \\ & \mathrm{~N} & 1551 & 1551\end{array}$

**. La correlación es significativa en el nivel 0,01 (bilateral).

Fuente: Elaboración propia con software SPSS, setiembre 2019.

Hay una relación directa y significativa entre las variables Puntaje de Examen de Ingreso y Rendimiento Académico de los estudiantes ingresantes a la
Universidad Nacional Jorge Basadre Grohmann, año académico 2018, Comprobada con una correlación de Pearson con un $\mathrm{p}=0,381$ y una significancia al $99 \%$.

Tabla 03. Estadísticos Inferencial (correlación Pearson) de las variables de Puntaje de Examen de Ingreso Admisión y el Rendimiento Académico de los estudiantes ingresantes a la UNJBG, año académico 2018.

$\begin{array}{llrr} & & \text { Puntaje de } & \\ & & \text { Examen de } & \text { Rendimiento } \\ & & \text { Ingreso } & \text { académico } \\ & & \text { ADMISION } & \text { UNJBG-2018 } \\ \text { Puntaje de Examen de } & \text { Correlación de Pearson } & 1 & 0,372^{* *} \\ \text { Ingreso ADMISION } & \text { Sig. (bilateral) } & & 0,000 \\ \text { Rendimiento académico } & \text { Correlación de Pearson } & 1054 & 1054 \\ \text { UNJBG-2018 } & \text { Sig. (bilateral) } & 0,372^{* *} & 1 \\ & \mathrm{~N} & 0,000 & 1054\end{array}$

**. La correlación es significativa en el nivel 0,01 (bilateral).

Hay una relación directa y significativa entre las variables Puntaje de Examen de Ingreso Admisión y Rendimiento Académico de los estudiantes ingresantes a la Universidad Nacional Jorge Basadre Grohmann, año académico 2018, Comprobada con una correlación de Pearson con un $\mathrm{p}=0,372$ y una significancia al $99 \%$.

Tabla 04. Estadísticos Inferencial (correlación Pearson) de las variables de Puntaje de Examen de Ingreso CEPU y el Rendimiento Académico de los estudiantes ingresantes a la UNJBG año académico 2018.

\begin{tabular}{llrr} 
& & & \multicolumn{2}{c}{$\begin{array}{c}\text { Rendimiento } \\
\text { académico UNJBG- } \\
\text { Puntaje de Examen de ingreso }\end{array}$} & $\begin{array}{c}\text { Puntaje de } \\
\text { Examen de CEPU }\end{array}$ & 2018 \\
CEPU & Correlación de Pearson & 1 &, $402^{* *}$ \\
& Sig. (bilateral) &, 000 \\
Rendimiento académico & $\mathrm{N}$ & 497 & 497 \\
UNJBG-2018 & Correlación de Pearson &, $402^{* *}$ & 1 \\
& Sig. (bilateral) &, 000 & 497
\end{tabular}

**. La correlación es significativa en el nivel 0,01 (bilateral).

Fuente: Elaboración propia con software SPSS, setiembre 2019. 
Hay una relación directa y significativa entre las variables Puntaje de Examen de Ingreso CEPU y Rendimiento Académico de los estudiantes ingresantes a la Universidad Nacional Jorge Basadre Grohmann, año académico 2018, Comprobada con una correlación de Pearson con un $\mathrm{p}=0,402$ y una significancia al $99 \%$.

\section{Verificación de las hipótesis}

\section{Hipótesis específicas}

Según la Tabla 03, existe una relación directa y significativa entre el puntaje examen de Admisión y el rendimiento académico de los estudiantes ingresantes de la Universidad Nacional Jorge Basadre Grohmann, año académico 2018, al tener la correlación de Pearson con un $\mathrm{p}=0.372$ y una significancia al $99 \%$.

b) Según la Tabla 04, existe una relación directa y significativa entre el puntaje examen de CEPU y el rendimiento académico de los estudiantes ingresantes de la Universidad Nacional Jorge Basadre Grohmann, año académico 2018, al tener la correlación de Pearson con un $\mathrm{p}=0.402$ y una significancia al $99 \%$.

\section{Hipótesis general}

Según la Tabla 02, existe una relación directa y significativa entre el puntaje examen de ingreso y el rendimiento académico de los estudiantes ingresantes de la Universidad Nacional Jorge Basadre Grohmann, año académico 2018, al tener la correlación de Pearson con un $\mathrm{p}=0,381$ y una significancia al $99 \%$.

\section{Modelo Matemático}

$$
y a b x=+
$$

$\mathbf{y}=$ Valor de la variable dependiente que se desea predecir

$\mathbf{a}=$ ordenada en el origen

$\mathbf{b}=$ pendiente o inclinación

$\mathbf{x}=$ valor que fijamos a la variable independiente

Tabla 05. Cálculo de R y R cuadrado

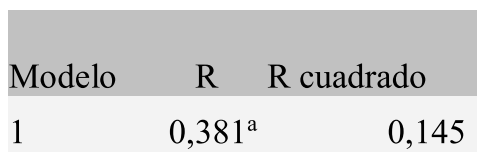

Fuente: Elaboración propia con software SPSS, setiembre 2019.

Gráfica 01: Diagrama de Dispersión de variables de estudio Puntaje de examen de ingreso y Rendimiento académico.

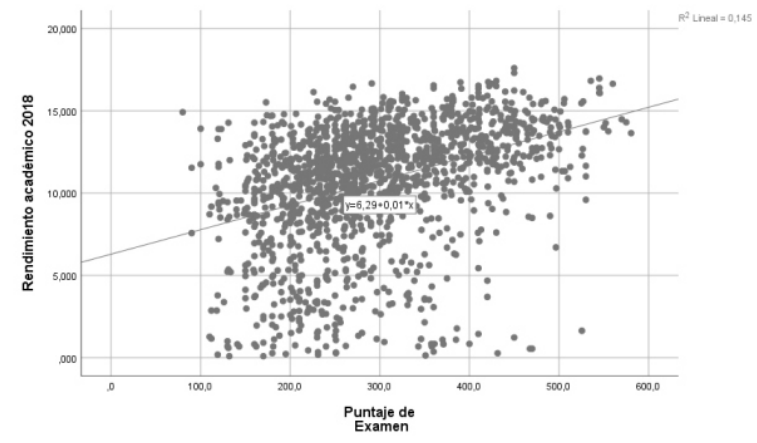

Fuente: Elaboración propia con software SPSS, setiembre 2019.

Tabla 06. Cálculo de coeficientes del modelo lineal

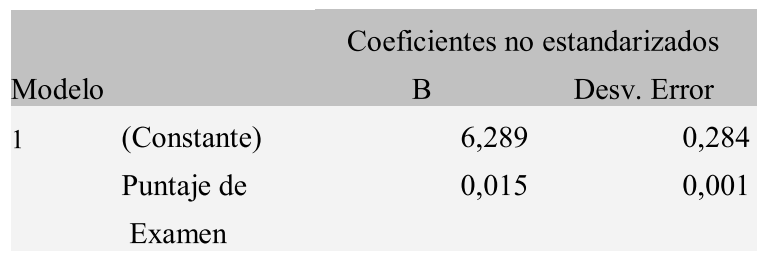

Fuente: Elaboración propia con software SPSS, setiembre 2019.

$$
\begin{array}{cc}
\qquad \begin{array}{c}
\mathrm{y}=\mathrm{a}+\mathrm{bx} \\
\mathrm{y}=6,289+0,015 \mathrm{x}
\end{array} \\
\mathbf{y}=\text { Rendimiento Académico } & 0.091 \leq \mathrm{y} \leq 17,591 \\
\mathbf{x}=\text { Puntaje de examen de ingreso } & 80,0 \leq \mathrm{x} \leq 580,5
\end{array}
$$

\section{Aplicación del Modelo Matemático}

¿Qué puntaje de rendimiento académico esperaría un estudiante con puntaje de examen de ingreso de 500 ?

$$
\begin{aligned}
& y=6.289+0.015(500) \\
& y=13.789
\end{aligned}
$$

\section{DISCUSIÓN}

A partir de los resultados hallados de la hipótesis general existe una relación directa y significativa entre el puntaje examen de ingreso y el rendimiento académico de los estudiantes ingresantes de la Universidad Nacional Jorge Basadre Grohmann, año académico 2018.

Estos resultados guardan relación con lo que sostienen Vergara \& Peredo (2017), quienes señalan que existe relación entre los instrumentos de selección de ingreso y el rendimiento académico posterior. Ello es acorde con lo que en este estudio se halla. 
Modelo matemático entre el puntaje de examen de ingreso y el rendimiento académico de los estudiantes ingresantes a la Universidad Nacional Jorge Basadre Grohmann, año académico 2018

En lo que respecta sobre los resultados de las hipótesis específicas. Existe una relación directa y significativa entre el Puntaje Examen de Ingreso, CEPU y el rendimiento académico de los estudiantes ingresantes de la Universidad Nacional Jorge Basadre Grohmann, año académico 2018. Con $\mathrm{p}=0.372$ y $\mathrm{p}=0.402$ con significancia al $99 \%$ respectivamente. Sin embargo, estos resultados no concuerdan con el estudio del autor Flores (2012), en lo que señala que la relación del rendimiento académico universitario con las modalidades de ingreso se encontró que los estudiantes obtuvieron promedios similares en el rendimiento académico universitario al margen de la modalidad de ingreso, en términos de promedio ponderado anual, la relación no fue estadísticamente significativa $(\mathrm{P}>0,05)$. Las modalidades de admisión parecen no ser un buen predictor del rendimiento académico; salvo el rendimiento escolar previo, una vez que el estudiante ingresa a la universidad se disipan algunas variables que los caracterizan durante la etapa escolar.

\section{CONCLUSIONES}

En estudiantes ingresantes de la Universidad Nacional Jorge Basadre Grohmann, año académico 2018, existe una relación positiva significativa entre el puntaje de examen de ingreso por Admisión y el rendimiento académico. Detectado, con una correlación de Pearson $\mathrm{p}=0,372$ y una significancia al $99 \%$.

En estudiantes ingresantes de la Universidad Nacional Jorge Basadre Grohmann, año académico 2018, existe una relación positiva significativa entre el puntaje de examen de ingreso por CEPU y el rendimiento académico. Detectado, con una correlación de Pearson $\mathrm{p}=0,402$ y una significancia al $99 \%$.

En estudiantes ingresantes de la Universidad Nacional Jorge Basadre Grohmann, año académico 2018; existe una relación positiva significativa entre el puntaje de examen de ingreso y el rendimiento académico. Detectado, con una correlación de Pearson $\mathrm{p}=0,372$ y una significancia al $99 \%$.

\section{REFERENCIAS BIBLIOGRÁFICAS}

BENÍTEZ, G. \& Ticona, L. (2006). Relación entre el rendimiento del examen de admisión y el académico, Tacna, 2001-2005. Ciencia \& Desarrollo 10, 15- 18.
CORTÉS, A. \& Palomar, J. (2008). El proceso de admisión como predictor del rendimiento académico en educación superior. Universitas Psychologica, 7 (1), 199-215.

CHÁveZ, M. \& Mendoza; J. (2006). Rendimiento académico de secundaria y su relación con el primer año de educación universitaria en la UN/JBG de Tacna, en el año. Revista Ciencia \& Desarrollo $1047-50$.

FLORES, M. (2012). Relación entre las modalidades de ingreso y el rendimiento académico de los estudiantes de estomatología de las cohortes 2008-2009 en una Universidad Privada. Revista Estomatológica Herediana 22(4) 210 215.

GARCÍA, C. (2005). Habilidades sociales, clima social familiar y rendimiento académico en estudiantes universitarios. Revista LIBERABIT 11 63-74.

NORMAS EDITORIALES, T. et al (2015). Efecto de la incorporación del ranking de notas en el proceso de admisión a las universidades chilenas. Pensamiento Educativo. Revista de Investigación Educacional Latinoamericana 52(1), 95-118.

RODRÍGUEZ Castro, M. \& Gómez López, V.M. (2010) Indicadores al ingreso en la carrera de medicina y su relación con el rendimiento académico. Revista de Educación Superior 39(1) 43-50.

RODRÍGUEZ C. \& Padilla, G. (2016), Trayectoria Escolar y Ranking: valoraciones $y$ estrategias institucionales en el nuevo escenario de selección universitaria. Revista Estudios Pedagógicos XLII, No 3: 313-326.

TREVIÑO Lozano, E. (2003). Relaciones entre el examen de ingreso y las calificaciones con la deserción y la utilidad de la tutoría para la permanencia en Fime de la UANL. Tesis de Grado, Universidad Autónoma de Nueva León, México.

VERGARA G. \& Peredo, H. (2017). Relación del desempeño académico de estudiantes de primer año de universidad en Chile y los instrumentos de selección para su ingreso. Revista Educación 41 (2) 2215-2644. 